\title{
Topological Interpretation of Rough Sets
}

\author{
Adam Grabowski \\ Institute of Informatics \\ University of Białystok \\ Akademicka 2, 15-267 Białystok \\ Poland
}

\begin{abstract}
Summary. Rough sets, developed by Pawlak, are an important model of incomplete or partially known information. In this article, which is essentially a continuation of [11, we characterize rough sets in terms of topological closure and interior, as the approximations have the properties of the Kuratowski operators. We decided to merge topological spaces with tolerance approximation spaces. As a testbed for our developed approach, we restated the results of Isomichi 13 . (formalized in Mizar in [14]) and about fourteen sets of Kuratowski [17] (encoded with the help of Mizar adjectives and clusters' registrations in [1] in terms of rough approximations. The upper bounds which were 14 and 7 in the original paper of Kuratowski, in our case are six and three, respectively.

It turns out that within the classification given by Isomichi, $1^{\text {st }}$ class subsets are precisely crisp sets, $2^{\text {nd }}$ class subsets are proper rough sets, and there are no $3^{\text {rd }}$ class subsets in topological spaces generated by approximations. Also the important results about these spaces is that they are extremally disconnected [15], hence lattices of their domains are Boolean.

Furthermore, we develop the theory of abstract spaces equipped with maps possessing characteristic properties of rough approximations which enables us to freely use the notions from the theory of rough sets and topological spaces formalized in the Mizar Mathematical Library [10.
\end{abstract}

MSC: $54 \mathrm{H} 10$ 68T37 03B35

Keywords: rough sets; rough approximations; Kuratowski closure-complement problem; topological spaces

MML identifier: ROUGHS_4, version: 8.1.03 5.23.1204

The notation and terminology used in this paper have been introduced in the following articles: [2], 22], [4], [9], 24], [20], 21], [5], 6], [14, [1], 25], [3], 7], [19], 27], [11, [12, 18], 26], 15], 28, 16], and [8]. 


\section{Preliminaries}

Now we state the proposition:

(1) Let us consider a set $T$ and a family $F$ of subsets of $T$. Then $F=$ $\{B$, where $B$ is a subset of $T: B \in F\}$.

Let $f$ be a function and $A$ be a set. We say that $A$ is $f$-closed if and only if (Def. 1) $A=f(A)$.

Let $X$ be a set and $F$ be a family of subsets of $X$. One can check that $F$ is $\cap$-closed if and only if the condition (Def. 2 ) is satisfied.

(Def. 2) Let us consider subsets $a, b$ of $X$. If $a, b \in F$, then $a \cap b \in F$.

We say that $F$ is union-closed if and only if

(Def. 3) Let us consider a family $a$ of subsets of $X$. If $a \subseteq F$, then $\bigcup a \in F$.

We say that $F$ is topology-like if and only if

(Def. 4) (i) $\emptyset, X \in F$, and

(ii) $F$ is union-closed and $\cap$-closed.

Let us observe that there exists a family of subsets of $X$ which is topologylike.

\section{Ordinary Properties of Maps}

Let $X$ be a set and $f$ be a function from $2^{X}$ into $2^{X}$. We say that $f$ is extensive if and only if

(Def. 5) Let us consider a subset $A$ of $X$. Then $A \subseteq f(A)$.

We say that $f$ is intensive if and only if

(Def. 6) Let us consider a subset $A$ of $X$. Then $f(A) \subseteq A$.

We say that $f$ is idempotent if and only if

(Def. 7) Let us consider a subset $A$ of $X$. Then $f(f(A))=f(A)$.

We say that $f$ is $\subseteq$-monotone if and only if

(Def. 8) Let us consider subsets $A, B$ of $X$. If $A \subseteq B$, then $f(A) \subseteq f(B)$.

We say that $f$ preserves $\cup$ if and only if

(Def. 9) Let us consider subsets $A, B$ of $X$. Then $f(A \cup B)=f(A) \cup f(B)$.

We say that $f$ preserves $\cap$ if and only if

(Def. 10) Let us consider subsets $A, B$ of $X$. Then $f(A \cap B)=f(A) \cap f(B)$.

Let $O$ be a function from $2^{X}$ into $2^{X}$. We say that $O$ is a preclosure if and only if

(Def. 11) $O$ is extensive and preserves $\cup$ and empty set.

We say that $O$ is closure if and only if

(Def. 12) $O$ is extensive and idempotent and preserves $\cup$ and empty set. 
We say that $O$ is a preinterior if and only if

(Def. 13) $O$ is intensive and preserves $\cap$ and universe.

We say that $O$ is an interior if and only if

(Def. 14) $O$ is intensive and idempotent and preserves $\cap$ and universe.

Let us observe that every function from $2^{X}$ into $2^{X}$ which preserves $U$ is also $\subseteq$-monotone and every function from $2^{X}$ into $2^{X}$ which preserves $\cap$ is also $\subseteq$-monotone.

One can verify that $\mathrm{id}_{2^{X}}$ is closure as a function from $2^{X}$ into $2^{X}$ and $\mathrm{id}_{2^{X}}$ is an interior as a function from $2^{X}$ into $2^{X}$.

One can check that there exists a function from $2^{X}$ into $2^{X}$ which is closure and interior.

Observe that every function from $2^{X}$ into $2^{X}$ which is closure is also a preclosure.

\section{Structural Part}

Let $T$ be a 1-sorted structure.

A map of $T$ is a function from $2^{\text {(the carrier of } T)}$ into $2^{\text {(the carrier of } T)}$. We consider 1stOpStrs which extend 1-sorted structures and are systems

$$
\text { 〈a carrier, a FirstOp〉 }
$$

where the carrier is a set, the FirstOp is a function from $2^{\text {(the carrier) }}$ into $2^{\text {(the carrier) }}$.

We consider 2ndOpStrs which extend 1-sorted structures and are systems

$$
\text { 〈a carrier, a SecondOp〉 }
$$

where the carrier is a set, the SecondOp is a function from $2^{\text {(the carrier) }}$ into $2^{\text {(the carrier) }}$.

We consider TwoOpStructs which extend 1stOpStrs and 2ndOpStrs and are systems

$$
\text { 〈a carrier, a FirstOp, a SecondOp〉 }
$$

where the carrier is a set, the FirstOp and the SecondOp are functions from $2^{\text {(the carrier) }}$ into $2^{\text {(the carrier) }}$.

Let $X$ be a 1 stOpStr. We say that $X$ has closure if and only if

(Def. 15) The FirstOp of $X$ is closure.

We say that $X$ has preclosure if and only if

(Def. 16) The FirstOp of $X$ is a preclosure.

Let $T$ be a topological space. Let us observe that ClMap $T$ is closure and IntMap $T$ is an interior and there exists a 1stOpStr which is non empty and has closure and every 1stOpStr which has closure has also preclosure. 
Let $X$ be a 1 stOpStr and $A$ be a subset of $X$. We say that $A$ is op-closed if and only if

(Def. 17) $\quad A=($ the FirstOp of $X)(A)$.

We say that $X$ has op-closed subsets if and only if

(Def. 18) There exists a subset $A$ of $X$ such that $A$ is op-closed.

One can check that there exists a 1stOpStr which has op-closed subsets.

Let $X$ be 1stOpStr with op-closed subsets. One can check that there exists a subset of $X$ which is op-closed.

Let $X$ be a 2 ndOpStr and $A$ be a subset of $X$. We say that $A$ is op-open if and only if

(Def. 19) $A=($ the SecondOp of $X)(A)$.

We say that $X$ has op-open subsets if and only if

(Def. 20) There exists a subset $A$ of $X$ such that $A$ is op-open.

Let us observe that there exists a $2 \mathrm{ndOpStr}$ which has op-open subsets.

Let $X$ be 2 ndOpStr with op-open subsets. Let us observe that there exists a subset of $X$ which is op-open.

Let $X$ be a 2 ndOpStr. We say that $X$ has interior if and only if

(Def. 21) The SecondOp of $X$ is an interior.

We say that $X$ has preinterior if and only if

(Def. 22) The SecondOp of $X$ is a preinterior.

Note that there exists a TwoOpStruct which has closure and interior.

\section{Merging With Topologies}

We consider 1TopStructs which extend 1stOpStrs and topological structures and are systems

$$
\text { 〈a carrier, a FirstOp, a topology〉 }
$$

where the carrier is a set, the FirstOp is a function from $2^{\text {(the carrier) into }}$ $2^{\text {(the carrier) }}$, the topology is a family of subsets of the carrier.

We consider 2TopStructs which extend 2ndOpStrs and topological structures and are systems

$$
\text { 〈a carrier, a SecondOp, a topology }
$$

where the carrier is a set, the SecondOp is a function from $2^{\text {(the carrier) }}$ into $2^{\text {(the carrier) }}$, the topology is a family of subsets of the carrier.

Let us observe that there exists a 1TopStruct which is non empty and strict and there exists a 2TopStruct which is non empty and strict.

Let $T$ be a 1TopStruct. We say that $T$ has properly defined topology if and only if 
(Def. 23) Let us consider an object $x$. Then $x \in$ the topology of $T$ if and only if there exists a subset $S$ of $T$ such that $S^{\mathrm{c}}=x$ and $S$ is op-closed.

Let $T$ be a 2 TopStruct. We say that $T$ has properly defined Topology if and only if

(Def. 24) Let us consider an object $x$. Then $x \in$ the topology of $T$ if and only if there exists a subset $S$ of $T$ such that $S=x$ and $S$ is op-open.

One can verify that there exists a 1 TopStruct which has closure and properly defined topology and there exists a 2TopStruct which has interior and properly defined Topology.

(2) Let us consider 1 TopStruct $T$ with properly defined topology and a subset $A$ of $T$. Then $A$ is op-closed if and only if $A$ is closed. Proof: If $A$ is op-closed, then $A$ is closed by [28, (3)]. If $A$ is closed, then $A$ is op-closed by [28, (3)].

Observe that every 1 TopStruct with properly defined topology which has preclosure is also topological space-like.

(3) Let us consider 2TopStruct $T$ with properly defined Topology and a subset $A$ of $T$. Then $A$ is op-open if and only if $A$ is open.

Note that every 2TopStruct with properly defined Topology which has preinterior is also topological space-like.

(4) Let us consider 1TopStruct $T$ with closure properly defined topology and a subset $A$ of $T$. Then (the FirstOp of $T)(A)=\bar{A}$. Proof: Set $f=$ the FirstOp of $T$. Consider $F$ being a family of subsets of $T$ such that for every subset $C$ of $T, C \in F$ iff $C$ is closed and $A \subseteq C$ and $\bar{A}=\cap F$. $\bar{A} \subseteq f(A)$ by (2), [18, (3)]. Define $\mathcal{P}$ [subset of $T] \equiv \$_{1} \in F$. Set $G=$ $\{f(B)$, where $B$ is a subset of $T: B \in F\}$. Define $\mathcal{T}=2^{\text {(the carrier of } T \text { ) }}$. Define $\mathcal{F}$ (element of $\mathcal{T})=f\left(\$_{1}\right)$. Define $\mathcal{G}($ element of $\mathcal{T})=\$_{1}$. For every element $B$ of $\mathcal{T}$ such that $\mathcal{P}[B]$ holds $\mathcal{F}(B)=\mathcal{G}(B) .\{\mathcal{F}(B)$, where $B$ is an element of $\mathcal{T}: \mathcal{P}[B]\}=\{\mathcal{G}(B)$, where $B$ is an element of $\mathcal{T}: \mathcal{P}[B]\}$ from [23, Sch. 6]. $F=G$. For every set $Z$ such that $Z \in G$ holds $f(A) \subseteq Z$.

\section{Introducing Rough Sets}

Let $R$ be a tolerance space. Let us note that $\operatorname{LAp}(R)$ is a preinterior and $\mathrm{UAp}(R)$ is a preclosure.

Let $R$ be an approximation space. Observe that $\operatorname{LAp}(R)$ is an interior and $\mathrm{UAp}(R)$ is closure.

Let $X$ be a set and $f$ be a function from $2^{X}$ into $2^{X}$. The functor GenTop $f$ yielding a family of subsets of $X$ is defined by

(Def. 25) Let us consider an object $x$. Then $x \in$ it if and only if there exists a subset $S$ of $X$ such that $S=x$ and $S$ is $f$-closed. 
Now we state the proposition:

(5) Let us consider a set $X$ and a function $f$ from $2^{X}$ into $2^{X}$. If $f$ is a preinterior, then GenTop $f$ is topology-like. Proof: Set $F=$ GenTop $f$. There exists a subset $S$ of $X$ such that $S=X$ and $S$ is $f$-closed. There exists a subset $S$ of $X$ such that $S=\emptyset$ and $S$ is $f$-closed. $F$ is $\cap$-closed. For every family $a$ of subsets of $X$ such that $a \subseteq F$ holds $\bigcup a \in F$ by [8, (74), (76)].

Let $C$ be a set, $I$ be a binary relation on $C$, and $f$ be a topology-like family of subsets of $C$. Observe that $\langle C, I, f\rangle$ is topological space-like and there exists a FR-structure which is topological space-like and non empty and has equivalence relation.

\section{On Sequential Closure and Frechet Spaces}

Let $T$ be a non empty topological space. The functor $\mathrm{Cl}_{\text {Seq }} T$ yielding a map of $T$ is defined by

(Def. 26) Let us consider a subset $A$ of $T$. Then it $(A)=\mathrm{Cl}_{\text {Seq }} A$.

One can verify that $\mathrm{Cl}_{\text {Seq }} T$ is a preclosure and there exists a non empty topological space which is Frechet.

Let $T$ be a Frechet non empty topological space. Note that $\mathrm{Cl}_{\text {Seq }} T$ is closure.

\section{Connections Between Closures and Approximations}

Let $T$ be a non empty FR-structure. We say that $T$ is Natural if and only if

(Def. 27) Let us consider a subset $x$ of $T$. Then $x \in$ the topology of $T$ if and only if $x$ is $(\operatorname{LAp}(T))$-closed.

We say that $T$ is naturally generated if and only if

(Def. 28) The topology of $T=$ GenTop LAp $(T)$.

Now we state the proposition:

(6) Let us consider a non empty FR-structure $T$. Suppose $T$ is naturally generated. Let us consider a subset $A$ of $T$. Then $A$ is open if and only if $\operatorname{LAp}(A)=A$.

Let us consider a non empty FR-structure $T$ and a non empty relational structure $R$.

Let us assume that the relational structure of $T=$ the relational structure of $R$. Now we state the propositions:

(7) $\operatorname{LAp}(T)=\operatorname{LAp}(R)$.

(8) $\operatorname{UAp}(T)=\operatorname{UAp}(R)$. 
One can verify that there exists a non empty FR-structure which is Natural and topological space-like and has equivalence relation and every non empty FR-structure with equivalence relation which is naturally generated is also topological space-like and there exists a non empty FR-structure which is naturally generated and topological space-like and has equivalence relation.

Let $T$ be a naturally generated non empty FR-structure with equivalence relation and $A$ be a subset of $T$. One can check that $\operatorname{LAp}(A)$ is open.

Let us consider a naturally generated non empty FR-structure $T$ with equivalence relation and a subset $A$ of $T$. Now we state the propositions:

(9) $\operatorname{LAp}(A)=\operatorname{Int} A$. Proof: $\operatorname{Int} A \subseteq \operatorname{LAp}(A)$ by [28, (22), (23)], [11, (24)].

(10) $A$ is closed if and only if $\operatorname{UAp}(A)=A$. Proof: If $A$ is closed, then $\mathrm{UAp}(A)=A$ by $(6),[11,(28)]$.

Let $T$ be a naturally generated non empty FR-structure with equivalence relation and $A$ be a subset of $T$. One can check that $\operatorname{UAp}(A)$ is closed.

Let us consider a naturally generated non empty FR-structure $T$ with equivalence relation and a subset $A$ of $T$. Now we state the propositions:

(11) $\operatorname{UAp}(A)=\bar{A}$. Proof: $\operatorname{UAp}(A) \subseteq \bar{A}$ by (10), [11, (25)], [19, (15)].

(12) $\operatorname{Bnd} \operatorname{Ap}(A)=\operatorname{Fr} A$. The theorem is a consequence of (11) and (9).

Let $T$ be a naturally generated non empty FR-structure with equivalence relation and $A$ be a subset of $T$. We identify $\operatorname{LAp}(A)$ with $\operatorname{Int} A$. We identify $\mathrm{UAp}(A)$ with $\bar{A}$. We identify $\operatorname{Int} A$ with $\operatorname{LAp}(A)$. We identify $\bar{A}$ with $\operatorname{UAp}(A)$. We identify $\operatorname{Fr} A$ with $\operatorname{BndAp}(A)$. We identify $\operatorname{BndAp}(A)$ with $\operatorname{Fr} A$.

\section{Isomichi Results Reuse}

Let us consider a naturally generated non empty FR-structure $T$ with equivalence relation and a subset $A$ of $T$. Now we state the propositions:

(13) $A$ is $1^{\text {st }}$ class if and only if $\operatorname{LAp}(\operatorname{UAp}(A)) \subseteq \operatorname{UAp}(\operatorname{LAp}(A))$.

(14) $A$ is $1^{\text {st }}$ class if and only if $\operatorname{UAp}(A) \subseteq \operatorname{LAp}(A)$.

(15) $A$ is $1^{\text {st }}$ class if and only if $A$ is exact. Proof: If $A$ is $1^{\text {st }}$ class, then $A$ is exact by [11, (14)], (14), [11, (13), (12)].

Let $T$ be a naturally generated non empty FR-structure with equivalence relation. Note that every subset of $T$ which is $1^{\text {st }}$ class is also exact and every subset of $T$ which is exact is also $1^{\text {st }}$ class.

Let us consider a naturally generated non empty FR-structure $T$ with equivalence relation and a subset $A$ of $T$. Now we state the propositions:

(16) $A$ is $2^{\text {nd }}$ class if and only if $\operatorname{LAp}(A) \subset \operatorname{UAp}(A)$.

(17) $A$ is $2^{\text {nd }}$ class if and only if $A$ is rough. $\operatorname{Proof}: \operatorname{LAp}(A) \neq \operatorname{UAp}(A)$ by [11, (13), (12)]. 
Let $T$ be a naturally generated non empty FR-structure with equivalence relation. Note that every subset of $T$ which is $2^{\text {nd }}$ class is also rough and every subset of $T$ which is rough is also $2^{\text {nd }}$ class.

Now we state the propositions:

(18) Let us consider a naturally generated non empty FR-structure $T$ with equivalence relation and a subset $A$ of $T$. Then $\overline{\operatorname{Int} A}$ and $\bar{A}$ are $\subseteq$ comparable.

(19) Let us consider a naturally generated non empty FR-structure $T$ with equivalence relation and a subset $A$ of $T$. Then $A$ is not $3^{\text {rd }}$ class.

Let $T$ be a topological space.

Observe that every naturally generated non empty FR-structure with equivalence relation is without 3rd class subsets and there exists a topological space which is without 3rd class subsets.

Let $T$ be a topological space and $A$ be a $1^{\text {st }}$ class subset of $T$. One can verify that Border $A$ is empty.

Let $T$ be a naturally generated non empty FR-structure with equivalence relation and $A$ be a subset of $T$. Note that $\bar{A}$ is open and $\operatorname{Int} A$ is closed and every naturally generated non empty FR-structure with equivalence relation is extremally disconnected.

\section{Reexamination of Kuratowski's 14 Sets for Approximation SPACES}

Let us consider a naturally generated non empty FR-structure $T$ with equivalence relation and a subset $A$ of $T$. Now we state the propositions:

(20) $\operatorname{Kurat7\operatorname {Set}}(A)=\{A, \bar{A}, \operatorname{Int} A\}$.

(21) $\overline{\overline{\operatorname{Kurat7Set}(A)}} \leqslant 3$. The theorem is a consequence of $(20)$.

(22) $\operatorname{Kurat14\operatorname {Set}}(A)=\left\{A, \operatorname{UAp}(A),(\operatorname{UAp}(A))^{\mathrm{c}}, A^{\mathrm{c}},(\operatorname{LAp}(A))^{\mathrm{c}}, \operatorname{LAp}(A)\right\}$.

$\overline{\overline{\operatorname{Kurat14} \operatorname{Set}(A)}} \leqslant 6$. The theorem is a consequence of $(22)$.

\section{REFERENCES}

[1] Lilla Krystyna Bagińska and Adam Grabowski. On the Kuratowski closure-complement problem. Formalized Mathematics, 11(3):323-329, 2003.

[2] Grzegorz Bancerek. Cardinal numbers Formalized Mathematics, 1(2):377-382, 1990.

[3] Grzegorz Bancerek. The ordinal numbers. Formalized Mathematics, 1(1):91-96, 1990.

[4] Grzegorz Bancerek and Krzysztof Hryniewiecki. Segments of natural numbers and finite sequences. Formalized Mathematics, 1(1):107-114, 1990.

[5] Czesław Byliński. Functions and their basic properties Formalized Mathematics, 1(1): 55-65, 1990.

[6] Czesław Byliński. Functions from a set to a set Formalized Mathematics, 1(1):153-164, 1990.

[7] Czesław Byliński. Partial functions Formalized Mathematics, 1(2):357-367, 1990. 
[8] Czesław Byliński. Some basic properties of sets. Formalized Mathematics, 1(1):47-53, 1990.

[9] Agata Darmochwał. Finite sets Formalized Mathematics, 1(1):165-167, 1990.

[10] Adam Grabowski. Automated discovery of properties of rough sets. Fundamenta Informaticae, 128:65-79, 2013. doi 10.3233/FI-2013-933

[11] Adam Grabowski. Basic properties of rough sets and rough membership function Formalized Mathematics, 12(1):21-28, 2004.

[12] Adam Grabowski. Relational formal characterization of rough sets. Formalized Mathematics, 21(1):55-64, 2013. doi 10.2478/forma-2013-0006.

[13] Yoshinori Isomichi. New concepts in the theory of topological space - supercondensed set, subcondensed set, and condensed set. Pacific Journal of Mathematics, 38(3):657-668, 1971.

[14] Magdalena Jastrzęska and Adam Grabowski. The properties of supercondensed sets, subcondensed sets and condensed sets. Formalized Mathematıcs, 13(2):353-359, 2005.

[15] Zbigniew Karno. The lattice of domains of an extremally disconnected space. Formalized Mathematics, 3(2):143-149, 1992.

[16] Artur Korniłowicz. On the topological properties of meet-continuous lattices Formalized Mathematics, 6(2):269-277, 1997.

[17] Kazimierz Kuratowski. Sur l'opération $\bar{A}$ de l'analysis situs. Fundamenta Mathematicae, 3:182-199, 1922.

[18] Beata Padlewska. Families of sets. Formalized Mathematics, 1(1):147-152, 1990.

[19] Beata Padlewska and Agata Darmochwał. Topological spaces and continuous functions Formalized Mathematics, 1(1):223-230, 1990.

[20] Bartłomiej Skorulski. First-countable, sequential, and Frechet spaces Formalized Mathematics, 7(1):81-86, 1998.

[21] Bartłomiej Skorulski. The sequential closure operator in sequential and Frechet spaces Formalized Mathematics, 8(1):47-54, 1999.

[22] Andrzej Trybulec. Enumerated sets Formalized Mathematics, 1(1):25-34, 1990.

[23] Andrzej Trybulec. Function domains and Frænkel operator. Formalized Mathematics, 1 (3):495-500, 1990.

[24] Andrzej Trybulec and Agata Darmochwał. Boolean domains Formalized Mathematics, 1 (1):187-190, 1990.

[25] Wojciech A. Trybulec and Grzegorz Bancerek. Kuratowski - Zorn lemma. Formalized Mathematics, 1(2):387-393, 1990.

[26] Zinaida Trybulec. Properties of subsets. Formalized Mathematics, 1(1):67-71, 1990.

[27] Edmund Woronowicz. Relations and their basic properties Formalized Mathematics, 1 (1):73-83, 1990.

[28] Mirosław Wysocki and Agata Darmochwał. Subsets of topological spaces Formalized Mathematics, 1(1):231-237, 1990.

Received March 31, 2014 